\title{
BMJ Open Quality Enhancing palliative care occupancy and efficiency: a quality improvement project that uses a healthcare pathway for service integration and policy development
}

To cite: Alshammary SA, Abuzied Y, Ratnapalan S. Enhancing palliative care occupancy and efficiency: a quality improvement project that uses a healthcare pathway for service integration and policy development. BMJ Open Quality 2021;10:e001391. doi:10.1136/ bmjoq-2021-001391

Received 13 February 2021 Accepted 10 October 2021
Check for updates

(C) Author(s) (or their employer(s)) 2021. Re-use permitted under CC BY-NC. No commercial re-use. See rights and permissions. Published by BMJ.

${ }^{1}$ Department of Palliative Care, Comprehensive Cancer Center, King Fahad Medical City, Riyadh, Saudi Arabia

${ }^{2}$ Department of Nursing, Rehabilitation Hospital, King Fahad Medical City, Riyadh,

Saudi Arabia

${ }^{3}$ Department of Pediatrics, University of Toronto Dalla Lana School of Public Health, Toronto, Ontario, Canada

Correspondence to

Yacoub Abuzied;

yabuzied2@gmail.com

\section{ABSTRACT}

This article described our experience in implementing a quality improvement project to overcome the bed overcapacity problem at a comprehensive cancer centre in a tertiary care centre. We formed a multidisciplinary team including a representative from patient and family support (six members), hospice care and home care services (four members), multidisciplinary team development (four members) and the national lead. The primary responsibility of the formulated team was implementing measures to optimise and manage patient flow. We used the plando-study-act cycle to engage all stakeholders from all service layers, test some interventions in simplified pilots and develop a more detailed plan and business case for further implementation and roll-out, which was used as a problem-solving approach in our project for refining a process or implementing changes. As a result, we observed a significant reduction in bed capacity from 35\% in 2017 to $13.8 \%$ in 2018 . While the original length of stay (LOS) was 28 days, the average LOS was 19 days in 2017 (including the time before and after the intervention), 10.8 days in 2018 (after the intervention was implemented), 10.1 days in 2019 and 16 days in 2020. The increase in 2020 parameters was caused by the COVID-19 pandemic, since many patients did not enrol in our new care model. Using a systematic care delivery approach by a multidisciplinary team improves significantly reduced bed occupancy and reduces LOS for palliative care patients.

\section{PROBLEM STATEMENT}

Palliative care is vital to enhance the quality of life for curative patients, seriously ill patients, patients in terminal stages or patients with considerable pain, including patients with cancer. In collaboration with other departments, the palliative care department (PCD) provides inpatient and outpatient care to optimise the target patients' well-being. Unfortunately, unexpected bed overcapacity by palliative care patients has been observed to highlight an urgent need for improvement.

The PCD is part of a comprehensive cancer centre (CCC) at King Fahad Medical City (KFMC), Riyadh, Saudi Arabia. KFMC is a
Ministry of Health tertiary care complex hospital with 1200 beds. It served around 30 000 inpatients and 500000 outpatients annually. The CCC is part of the national cancer strategy and is considered a primary ministry of a health reference centre for patients with cancer from all Saudi Arabia regions. The centre covers haematology, bone and marrow transplant and medical oncology for adult and paediatric patients, in addition to radiation therapy and palliative treatment. The total bed capacity for palliative care in CCC represents 87 beds.

Based on the available logistic patient's information, the PCD established a plan to have an occupancy rate of around 10\% of all beds in CCC for palliative care patients. However, we observed that palliative care patients occupied $35 \%-50 \%$ of all CCCs, representing around $300 \%$ of allocated to palliative care patients. This bed overcapacity leads to hospital-wide logistic and clinical burdens. It contributed to an increased burden on human resources, leading to staff shortage and increasing healthcare providers' workload. Besides, bed overcapacity disrupts other services such as the emergency department, internal medicine and surgery by displaced patients with cancer and unplanned emergency room (ER) visits. Concurrently, we observed a prolonged length of stay (LOS) for palliative care patients with a LOS average of 28 days. Figure 1 shows the problem highlights and critical challenging.

The quality team at PCD realised the burden of this problem. We implemented a quality improvement project to reduce the overbed capacity for palliative care patients from $35 \%$ in January 2017 by $10 \%$ by May 2018 in CCC. We aimed to reduce palliative care patients' average LOS (ALOS) from 28 days in January 2018 by 20\% in May 2018. 


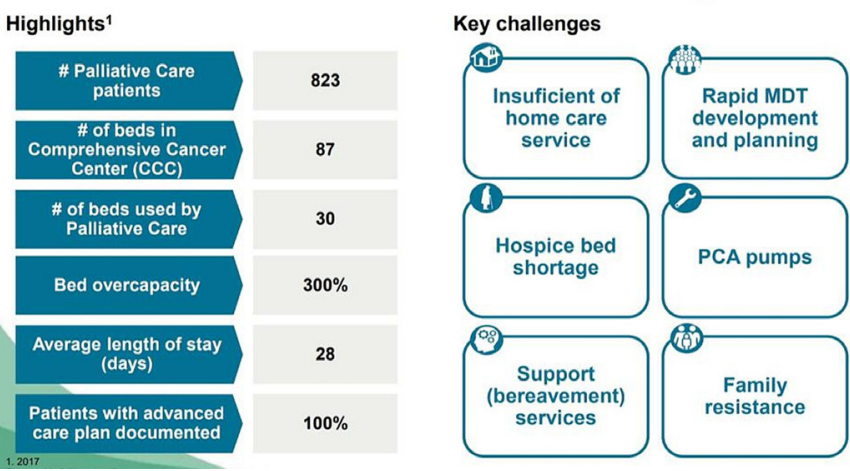

Figure 12017 statistics and key challenges. MDT, multidisciplinary team; PCA, patient control analgesia.

\section{BACKGROUND}

Palliative care aims to improve the quality of life for both patients and families. It focuses on preventing and mitigating suffering from physical, psychological, social or spiritual symptoms associated with life-threatening conditions. ${ }^{1}$ Palliative care is provided by a multidisciplinary team, as it requires integration and management for multiple internal and external services and entities.

Integration of palliative care into public health systems and other care levels is an ethical responsibility, ${ }^{2}$ yet more than $85 \%$ of patients globally who need palliative care cannot reach it. ${ }^{1}$ Improving access to palliative care requires increasing the capacity of an existing programme and developing healthcare programmes. More attention should be given to understanding and organising palliative care, training healthcare providers and providing public education. Decision-makers should address the multidisciplinary healthcare professions, policy and procedures, required resources, required knowledge and skills and education. ${ }^{13}$

Ignoring the current state resources, prioritising bed allocation, targeted stakeholders or the required skills and knowledge will lead to misusing or overusing the resources. For example, many of the available beds are occupied by patients requiring alternative care levels or receiving in-hospital care when not needed (unnecessarily occupying of bed).

Struggling with bed congestion (overcapacity) is a global problem for the healthcare system. Usually is reflected by mismatches between capacity and demand while providing care. ${ }^{45}$ It contributed to a chronic bed shortage, staff shortage and increased the burden on other departments, leading to overcrowded, delay in care and financial consequences, insufficient coordination between departments and prolonged LOS. ${ }^{6-8}$

Healthcare management identifies the way a healthcare facility is organised and coordinated. It contributed to finding solutions in different healthcare areas, including bed capacity and LOS. For example, management of bed overcapacity varied according to specified problems or departments (one size does not fit all). The different intervention has been applied to manage overcapacity problems. Examples of these strategies include the overcapacity management model, ${ }^{4}$ dynamic inpatient bed management by reducing non-emergency department (ED) admissions, ${ }^{8}$ full capacity protocol, ${ }^{9}$ diagnostictherapeutic-assistance path, ${ }^{10-12}$ discharge lounge, ${ }^{13}$ bed huddles ${ }^{14}$ reopening previously closed beds or adding new beds ${ }^{5}$ or forecasting modelling framework. ${ }^{15}$ These interventions focused on expanding, reducing the boarding time, reducing the LOS, improving patients' flow, free up available spaces or forecasting modelling.

Palliative care aims to support people with complex needs by providing care by different specialties. Using a multidisciplinary team and integrating a structured method to assess and treat palliative care patients is essential to ensure consistency and a systematic approach in delivering palliative care. ${ }^{16}$ We believe that a systematic care delivery approach for improving an existing capacity is necessary to provide the proper care, right place and right time. There was a real need to improve palliative care bed occupancy and efficiency in order to provide proper care for all patients and the projected proposal was strongly agreed upon and supported by the leaders, where this will improve palliative care in general, mainly bed occupancy and cost-effectiveness. Where our aim was to reduce the overbed capacity for palliative care patients in CCC. Also, we aimed to reduce the ALOS of palliative care patients, as well as their cost-effectiveness. From the start, we established a plan that included frequent multidisciplinary meetings to ensure the success, development and sustainability of our project, as well as an evaluation process of weekly challenges, obstacles and accomplishments. The assumptions were that by having an organised team and support from the institution and leader, the outcomes would be as predicted and ideal. This article described our experience implementing a quality improvement project to overcome the bed overcapacity problem at a CCC in a tertiary care centre. This article followed the Standards for Quality Improvement Reporting Excellence (SQUIRE) guidelines. ${ }^{17}$ The SQUIRE guidelines provide a framework to report on new insights into how healthcare is improved. They are intended for reports describing healthcare workers' quality, safety and value at the system level.

\section{MEASUREMENTS}

For this project, we collected data before and after the initiation of the quality improvement project. We calculated the CCC bed occupancy rate by palliative care patients monthly. Then, we divided the mean number of monthly beds occupied by palliative care patients by the total number of all CCC beds ( 87 beds). We also calculated the entire LOS days and the average LOS for palliative care patients in CCC by reviewing the date of hospital admissions and hospital discharges. Also, we calculated the average monthly number of unplanned ER visits by palliative care patients (table 1). 
Table 1 Unplanned emergency room visits by palliative care patients

\begin{tabular}{lclcc}
\hline & $\mathbf{2 0 1 7}$ & $\mathbf{2 0 1 8}$ & $\mathbf{2 0 1 9}$ & $\mathbf{2 0 2 0}$ \\
\hline January & 5 & 5 & 8 & 7 \\
February & 8 & 5 & 7 & 4 \\
March & 5 & 9 & 7 & 6 \\
April & 9 & 5 & 4 & 11 \\
May & 10 & 9 & 2 & 9 \\
June & 9 & 7 & 11 & 13 \\
July & 9 & 5 & 8 & 6 \\
August & 9 & 6 & 9 & 9 \\
September & 13 & 4 & 8 & 10 \\
October & 6 & 5 & 5 & 12 \\
November & 5 & 3 & 9 & 7 \\
December & 7 & 4 & 9 & 7 \\
Annual average & 7.92 & 5.58 & 7.25 & 8.42 \\
\hline & & & &
\end{tabular}

ER visits of 2020 are mostly patients who were not recruited into our new model of care. Therefore, we expect this indicator to lag behind 8-12 months.

We calculated the cost of care for admitted palliative care patients by multiplying the total number of patient's days by the estimated cost per patient. To meet our project goals for reducing bed overcapacity and LOS for palliative care patients, we planned to start measuring from January 2017 and continue counting until December 2020 (1000 days for PC transformation). Measuring project indicators over a long time will allow us to observe the improvement and observe its sustainability. Data were collected by trained staff from the quality improvement team at PCD.

Baseline measurement showed that the average number of beds used by palliative care patients in CCC was 30, representing $35 \%$ of CCC bed occupancy. This rate exceeds three times the planned rate of $10 \%$ of CCC beds by palliative care patients. The baseline average LOS was 28 days. The number of unplanned ER visits was 7.9 in 2017. The cost of care for admitted palliative care patients in CCC was US\$18 170000 .

\section{Patient and public involvement}

Patients were not specifically included in the analysis since all statistics were gathered from the department's database, including data on admissions and discharges during their stay at the hospital if their permission is not necessary as the approval received from the department chair.

\section{Design}

Understanding the system's complexity and its interaction is crucial for successfully implementing any quality improvement project. ${ }^{18}$ Palliative care is a complex setting requiring a multidisciplinary team and a systematic approach to assess and treat patients. ${ }^{16}$ Similarly, solving problems and improving the palliative care setting processes required a systematic approach involving all stakeholders. Therefore, we initiated a future state pathway map for the palliative care process (figure 2). We formed a multidisciplinary team, including a representative from patients and family support (six members), hospice care and home care services (four members), multidisciplinary team development (four members) and the national lead. The primary responsibility of the formulated team was implementing measures to optimise and manage patient flow.

To measure our quality improvement project's effectiveness on the process, we implemented a pre and post quasi-experimental design, in which the observations are made before and after the intervention. We analysed the

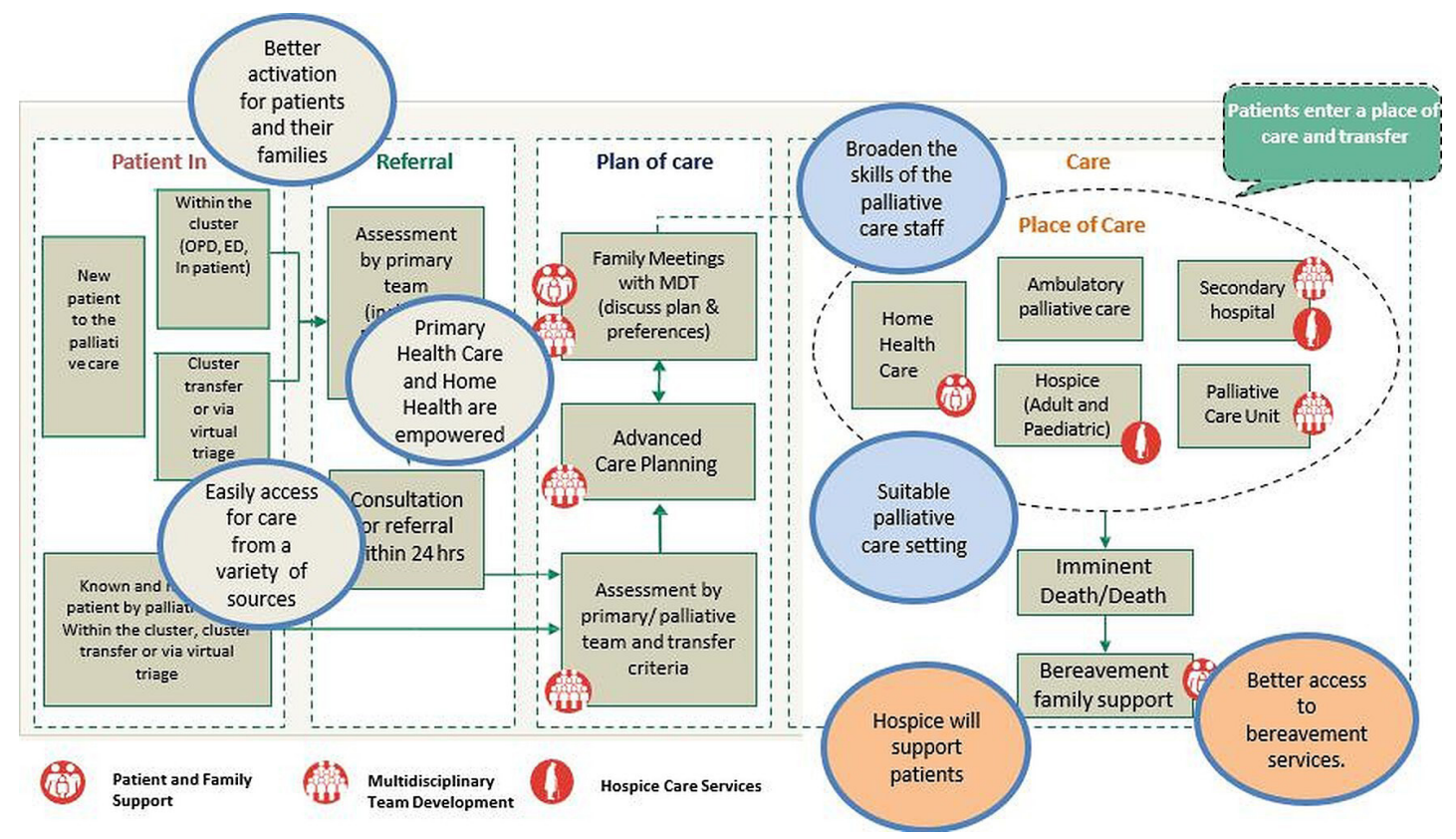

Figure 2 Future state pathway map-palliative care. 
General Actions

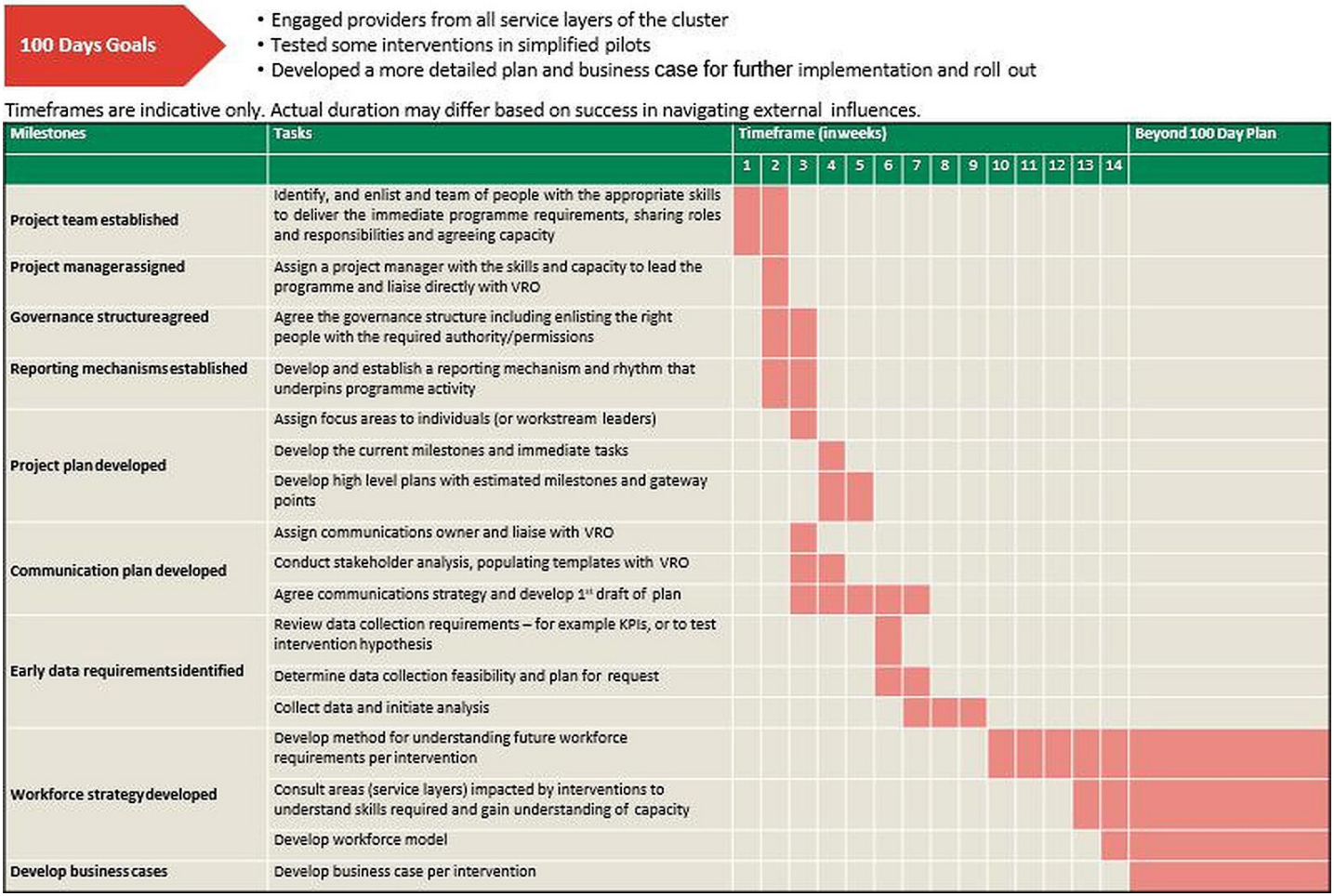

Figure 3 Implementation plan. KPls, key performance indicators; VRO, vision realisation office.

data over time and presented them using run charts and tables. To ensure that the improvement is related to the intervention, we measured the data for a long time; more specifically, we measured the data in 4 years (2017-2020). To make our intervention sustainable, we developed and implemented relevant tools, policies and procedures. We set goals for a 100-day plan to complete our intervention (figures 3 and 4). In addition, we calculated and compared the cost of care.

\section{Strategy}

Our smart aim was to reduce the overbed capacity for palliative care patients from $35 \%$ in January 2018 to $10 \%$ by May 2018 in CCC. Also, we aimed to reduce the ALOS

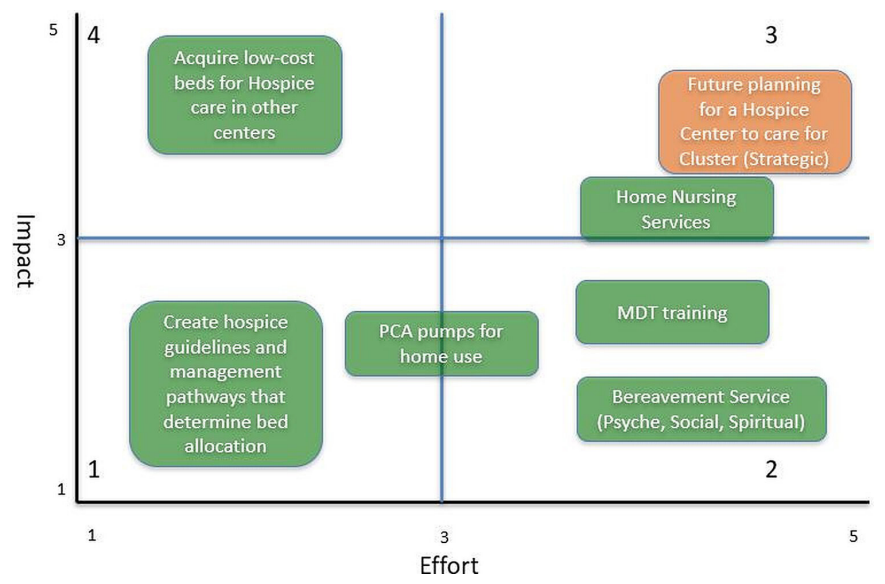

Figure 4 Effort impact diagram. MDT, multidisciplinary team; PCA, patient control analgesia. of palliative care patients from 28 days in January 2018 by $20 \%$ in May 2018. Plan-do-study-act cycle was used as a problem-solving approach in our project for refining a process or implementing changes to enlist the participation of all stakeholders from all service tiers. Some interventions were tested in simpler pilots and a more comprehensive strategy and business case was developed for future implementation and roll-out. We set goals for a 100-day plan to complete our intervention (implementation of the future state pathway, as well as policies and procedures that support and stabilise the pathway). At the end of 100 days, the team had:

- Engaged all stakeholders from all services.

- Tested some interventions in simplified pilots.

- Developed a more detailed plan and business case for further implementation and roll-out.

Our initial step was to engage multiple stakeholders from different layers. As a result, for example, we have begun to impact engagement with and the effectiveness of family meetings positively.

- Identify and enlist a team of people with the appropriate skills to deliver the immediate programme requirements, share roles and responsibilities and agree on capacity.

- Assign a project manager with the skills and capacity to lead the programme and liaise directly with the vision realisation office (VRO).

- Agree on the governance structure, including enlisting the right people with the required authority/ permissions. 


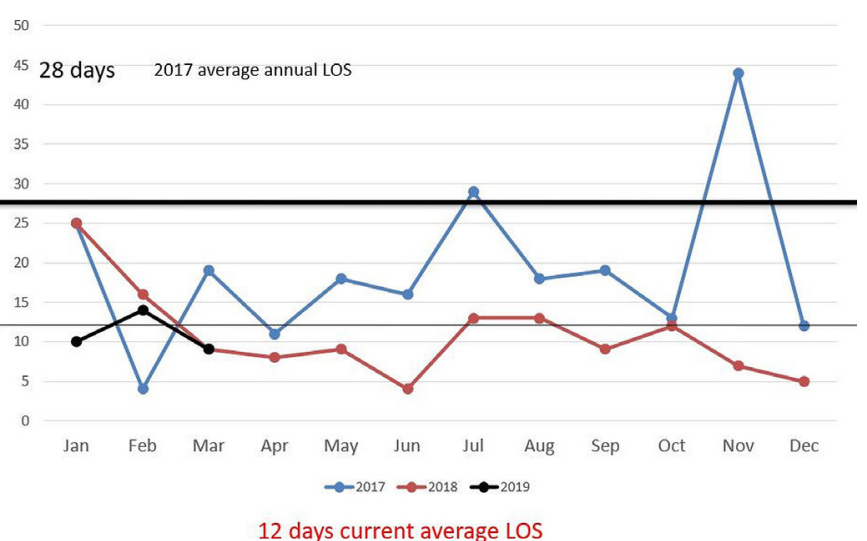

Figure 5 Length of Stay (LOS) run chart.

- Develop and establish a reporting mechanism and rhythm that underpins programme activity.

- Assign focus areas to individuals (or workstream leaders).

- Develop current milestones and immediate tasks.

- Develop high-level plans with estimated milestones and gateway points.

- Assign communications owner and liaise with VRO.

- Conduct stakeholder analysis, populating templates with VRO.

- Agree on communications strategy and develop a first draft of the plan.

- Review data collection requirements-for example, key performance indicators (KPIs) or to test intervention hypothesis.

\section{RESULTS}

Our primary outcome measures were the average number of beds occupied by palliative care patients in CCC and their average LOS. We also calculated the average number of unplanned ER visits and the cost of care. We observed a significant reduction in bed capacity from $35 \%$ in 2017 to $13.8 \%$ in 2018. The LOS was 28 days before implementing the intervention. The average LOS was 19 days in 2017 (before and after the intervention), 10.8 days in 2018 (after implementing the intervention), 10.1 days in 2019 and 16 days in 2020. These data are presented in a run-chart (figure 5). The average number of unplanned visits decreased from 7.9 in 2017 to 5.5 in 2018 and 7.3 in 2019. However, it was increased in 2020 to 8.7 (figure 5). The total annual cost was reduced by $69 \%$, from US $\$ 19642000$ to US\$5 438600 .

Controlling the sustainability of the process was evidenced by the preservation of reducing all measures over the years. However, due to the COVID-19 pandemic, many patients were not recruited into our new care model. Because of this reason, the measures of 2020 were increased in comparison to previous years. Therefore, we expect this indicator to lag behind 8-12 months.

\section{Lessons and limitations}

The primary objective of this project was to enhance palliative care occupancy and efficiency. The outcome showed significant improvement as the average of occupied beds by palliative care patients reduced from $35 \%$ to $13.8 \%$ in CCG.

Our quality improvement project uses a systematic care delivery approach that requires the interactions of a multidisciplinary team. This approach significantly reduced the LOS for palliative care patients and reduced the annual cost of care. Using a systematic way to solve problems or improve palliative care settings is essential as palliative care requires multiple stakeholders' cooperation and interactions. ${ }^{16}$ In addition, previous studies highlighted the importance of proactive and multidisciplinary care in reducing LOS and hospital expences. ${ }^{19}$

An altered discharging plan can increase LOS and increase the bed occupancy rate where the bed utilisation will be affected, hence increasing the cost. ${ }^{20}$ The process of discharging patients is complicated as it requires coordination from a multidisciplinary group, including physicians, nurses, ancillary service staff, patients, and families. This study provides a set of measures that are within the hospital's control to improve LOS. This project was initiated to decrease bed overcapacity, reduce the LOS, and provide the tools to develop and implement relevant policies and procedures.

Our results should be interpreted in light of their strengths and weaknesses. The studies that discussed quality improvement projects for bed overcapacity are limited. This article discussed applying a systematic care delivery approach model to promote palliative care's capacity and efficiency. Our results emphasised that using a systematic approach by engaging a multidisciplinary team improved bed capacity, LOS and the cost of care. Also, our results assured that this approach is sustainable over time. However, plans should be set to overcome emergent challenges such as what happened during the COVID-19 pandemic.

The main limitation is represented in the nature of the pre and post quasi-experimental design. Thus, it is challenging to know the exact responsible factor for process improvement, mainly when using interventions with multiple combinations. Besides, it is not easy to control various confounders over time. ${ }^{21}$ However, measuring the intervention's effectiveness over a long time may reduce the burden of these limitations, especially in continuous improvement.

\section{CONCLUSION}

A systematic care delivery approach by including a multidisciplinary team improves a palliative care setting's capacity and efficiency. This approach significantly enhances bed overcapacity and reduces LOS. Providing tools, policies and procedures will help in the sustainability of the project over time. Due to the significant project outcome, sharing this intervention will help measure, validate and improve it when used by others. 
Acknowledgements The authors would like to thank King Fahad Medical City through the research centre for technical support.

Contributors The research team designed and led the study's design and the article's preparation; also carried out data extraction and analysis and carried out a systematic review of the literature for data retrieval. The final version of this manuscript was read and approved by all authors. Before submission, all listed authors have approved the manuscript, including the names and data included and the graphs. All of the writers got input and helped shape the research, review and manuscript. SAA and SR designed and directed the presented study. YA and SAA wrote the manuscript with input from SAA. All the authors contributed to sample preparation. YA and SAA collected the data. YA performed the calculations and designed the figures. SAA supervised the project. Both SAA and YA contributed to the final version of the manuscript.

Funding The authors have not declared a specific grant for this research from any funding agency in the public, commercial or not-for-profit sectors.

Competing interests None declared.

Patient and public involvement Patients and/or the public were not involved in the design, or conduct, or reporting, or dissemination plans of this research.

Patient consent for publication Not applicable.

Ethics approval The Institutional Review Board at King Fahad Medical City gave ethical approval for this study. The authors used primary data collected by the research team. The chairperson of the respective departments granted approval for collecting data.

Provenance and peer review Not commissioned; externally peer reviewed.

Data availability statement Data are available upon reasonable request.

Open access This is an open access article distributed in accordance with the Creative Commons Attribution Non Commercial (CC BY-NC 4.0) license, which permits others to distribute, remix, adapt, build upon this work non-commercially, and license their derivative works on different terms, provided the original work is properly cited, appropriate credit is given, any changes made indicated, and the use is non-commercial. See: http://creativecommons.org/licenses/by-nc/4.0/.

ORCID iD

Yacoub Abuzied http://orcid.org/0000-0003-1388-4549

\section{REFERENCES}

1 Palliative care, 2020. Available: https://www.who.int/health-topics/ palliative-care [Accessed 20 Dec 2020].

2 Berendt J, Stiel S, Simon ST, et al. Integrating palliative care into comprehensive cancer centers: consensus-based development of best practice recommendations. Oncologist 2016;21:1241-9.

3 O'Mahony S, Levine S, Baron A, et al. Palliative workforce development and a regional training program. Am J Hosp Palliat Care 2018;35:138-43.
4 Kreindler SA, Star N, Hastings S, et al. "Working Against Gravity": The Uphill Task of Overcapacity Management. Health Serv Insights 2020;13:117863292092998.

5 Bazzoli GJ, Brewster LR, May JH, et al. The transition from excess capacity to strained capacity in U.S. hospitals. Milbank $Q$ 2006;84:273-304.

6 Abuzied Y, Maymani H, AlMatouq B, et al. Reducing the length of stay by enhancing the patient discharge process: using quality improvement tools to optimize Hospital efficiency. Global J Qual Saf Healthcare 2021:4:44-9.

7 Long EF, Mathews KS. The boarding patient: effects of ICU and hospital occupancy surges on patient flow. Prod Oper Manag 2018;27:2122-43.

8 Pines JM, Batt RJ, Hilton JA, et al. The financial consequences of lost demand and reducing boarding in hospital emergency departments. Ann Emerg Med 2011;58:331-40.

9 Alishahi Tabriz A, Birken SA, Shea CM, et al. What is full capacity protocol, and how is it implemented successfully? Implement Sci 2019;14:73.

10 Ricciardi C, Fiorillo A, Valente AS, et al. Lean six sigma approach to reduce Los through a diagnostic-therapeutic-assistance path at A.O.R.N. A. Cardarelli. TQM 2019;31:657-72.

11 Scala A, Ponsiglione AM, Loperto I, et al. Lean six sigma approach for reducing length of hospital stay for patients with femur fracture in a university hospital. Int J Environ Res Public Health 2021;18:2843.

12 Improta G, Luciano MA, Vecchione D. Management of the diabetic patient in the diagnostic care pathway. European Medical and Biological Engineering Conference, 2020:784-92.

13 Hernandez N, John D, Mitchell J. A reimagined discharge lounge as a way to an efficient discharge process. BMJ Qual Improv Rep 2014;3. doi:10.1136/bmjquality.u204930.w2080. [Epub ahead of print: 31 Jul 2014].

14 McBeth CL, Durbin-Johnson B, Siegel EO. Interprofessional Huddle: One Children's Hospital's Approach to Improving Patient Flow. Pediatr Nurs 2017;43:71-6.

15 Ordu M, Demir E, Tofallis C. A comprehensive modelling framework to forecast the demand for all hospital services. Int J Health Plann Manage 2019;34:e1257-71.

16 Hudson P, Collins A, Bostanci A, et al. Toward a systematic approach to assessment and care planning in palliative care: a practical review of clinical tools. Palliat Support Care 2016;14:161-73.

17 Ogrinc G, Mooney SE, Estrada C, et al. The Squire (standards for quality improvement reporting excellence) guidelines for quality improvement reporting: explanation and elaboration. Qual Saf Health Care 2008;17:113-32.

18 Toulany A, McQuillan R, Thull-Freedman JD, et al. QuasiExperimental designs for quality improvement research. Implementation Sci 2013;8:S3.

19 Zou J, Xu X, Wang D, et al. [The impacts of the multidisciplinary team model on the length of stay and hospital expenses of patients with lung cancer]. Zhonghua Jie He He Hu Xi Za Zhi 2015;38:370-4.

20 Lin C-J, Cheng S-J, Shih S-C, et al. Discharge planning. Int J Gerontol 2012;6:237-40.

21 Ambroggio L, Schondelmeyer A, Hoefgen E, et al. Quality improvement feature series article 4: advanced designs for quality improvement studies. J Pediatric Infect Dis Soc 2017;41:335-7. 